\title{
Updating an object's orientation and location during nonvisual navigation: A comparison between two processing modes
}

\author{
MICHEL-ANGE AMORIM \\ Collège de France, Paris, France \\ STEFAN GLASAUER \\ University of Munich, Munich, Germany \\ and \\ KATTY CORPINOT and ALAIN BERTHOZ \\ Collège de France, Paris, France
}

\begin{abstract}
In the present study, we compared the effects of two processing modes on the updating of the location and orientation of a previously viewed object in space during a guided walk without vision. In Experiment 1, in order to measure the error for initial perception of object's orientation, 12 subjects rotated a miniature model until it matched the memorized orientation of its counterpart object in space. In Experiment 2, they attempted either to keep track of the object continuously (in the object-centered $[\mathrm{OC}]$ task) or to estimate the object's perspective only at the terminal vantage point given the trajectory they walked (in the trajectory-centered [TC] task). Subjects indicated the location of the object by facing it, and then rotated the model in order to indicate its orientation from the new vantage point. Results showed that, with respect to the TC mode, the OC mode induced a slowdown of the subjects' self-paced locomotion velocity for both linear and angular movements, and a decrease of the latencies as well as smaller absolute errors for the orientation-of-the-object response. Mean signed errors on object's orientation were equivalent for both processing modes, suggesting that the latter induced different allocations of processing resources on a common representation of space updated by "path integration."
\end{abstract}

Mentally updating the location and orientation of a previously seen distant sniper while walking in complete darkness, a potentially crucial task, can be performed in two ways. We can imagine the passing scene continuously, paying less attention to the actual path along which we are walking (Loarer \& Savoyant, 1991; Rieser, 1990). Or, we can attend to the path - as if wanting to retrace our steps exactly - and fail to attend to the scene around the path, except that, at the terminus of the path, we might "reconstruct" or "infer" the new directions of the parts of the surrounding scene (Huttenlocher \& Presson, 1973; Levine, Jankovic, \& Palij, 1982). The prerequisite for both kinds of computation is a process called "path integration" (Mittelstaedt \& Glasauer, 1991; Mittelstaedt \& Mittelstaedt, 1980), which uses the available vestibular,

This research was supported by a doctoral grant from the Centre National d'Etudes Spatiales to M.-A.A., and by a grant from Axe Thématique Espace of the Cogniscience Programme. We thank M. Ehrette and M. Loiron for the technical assistance they provided, respectively, in the stimuli fabrication and the setup of the wireless transmission. We also thank J. Cutting, R. Grasso, L. Krueger, E. Loarer, J. Loomis, M. May, S. Wiener, and three anonymous reviewers for their comments on a first draft of this paper. Correspondence should be addressed to M.-A. Amorim, Laboratoire de Physiologie de la Perception et de l'Action, Collège de France, CNRS, 11 place Marcelin Berthelot, F-75005 Paris, France (e-mail: amorim@ccr.jussieu.fr). kinesthetic, and motor command information in order to maintain self-orientation and position during locomotion in the temporary absence of vision (Loomis et al., 1993). Previous studies have shown that such a process demands central processing capacity since it can be interfered with by a concurrent task, either before (i.e., when encoding a targeted location; Corlett, Kozub, \& Quick, 1990) or during (Böök \& Gärling, 1980) the blindfolded walk. Moreover, effects of practice and information load also indicate that the processing of locational information is effortful (Lindberg \& Gärling, 1983) rather than automatic, and that it is related to visuospatial imagery (Klatzky et al., 1990; Thorndyke \& Hayes-Roth, 1982). However, to our knowledge, no study has addressed the specific issue of the effect of two processing modes on nonvisual navigation in terms of processing resources allocated differently to relevant spatial information in order to fulfill the task rather than in terms of interfering concurrent tasks that would never allow a correct response to emerge anyway.

Although there exists a literature on solution strategies for a few spatial tasks (see Lohman, 1988, for details), this kind of study is certainly lacking in the field of nonvisual navigation. It is our goal to show that there are at least two different modes, or strategies, for processing information centrally in order to update spatial information while walking without vision, and to describe their effects in 
terms of processing time and error. In addition, in contrast to previous studies on blind walking, which have mainly been concerned with the updating of the location of single objects or configuration of objects (Amorim, Loomis, \& Fukusima, 1995; Easton \& Sholl, 1995; Loomis et al., 1993; Rieser, 1989; Rieser \& Rider, 1991), here we examine the updating of both the location and the orientation of a previously viewed object in geographic space.

Why should spatial information be processed differently? The transformations occurring in the represented space during walking without vision, after the memorization of the initial attributes of an object or a scene, are the result of both path integration and allocation of attention. In an emergency, we may be willing to privilege speed over accuracy, thereby allocating more attention to the path we walk rather than updating the whole scene that surrounds us (see next section, Issue 2). Both modes surely operate on the same represented space, although the spatial information to which processing resources are allocated is not the same. However, it is not expected that two interchangeable processing modes have dramatically distinct effects on the spatial updating of performance, as measured by errors. Since both processing modes share the same initial input from the path integration process, the errors for each mode should not differ significantly when the location and orientation of an object are being updated, although the variability in the errors may be different. Nevertheless, the two processing modes might differ by their effect on the locomotion velocity and response latency. By analogy, one may retrieve as accurately the hypotenuse of a right isosceles triangle either by calculus or by drawing and measuring it, but one process might lead to a faster response than the other.

\section{THE PRESENT STUDY}

Landmarks are the initial places learned in an environment (Golledge, 1987). Locating our position on the basis of a directionally polarized object is quite a common task in our everyday life: For example, it is typical to retrieve our position in a town by watching the direction of landmarks such as panels, statues, buildings, and so on. Therefore, we decided to investigate the updating of both an object's location and orientation during locomotion in the temporary absence of vision, that is, nonvisual navigation.

In order to compare the characteristics and effects of two processing modes on the updating of the location and orientation of an object (i.e., of its attributes), we had to find methodological answers to (1) control for the initial encoding of the object's attributes, (2) constrain observers to adopting each processing mode, and (3) separate the sources of errors in the observer's response concerning the location and orientation of the object.

Each of these issues is considered here.

1. What were the available cues for encoding the initial attributes of the object? The object we used was a 3dimensional uppercase letter $F$. The selection of this object was based on its peculiar symmetry properties, namely its clear directional polarization. The attributes of this ob- ject inside the geographic space may be defined as follows: its vertical bar indicates its location, and its two horizontal bars indicate the direction it points in space (i.e., its orientation). Observers initially viewed the attributes of this to-be-updated object under a reduced-cue condition, that is, the object's borders were glowing in complete darkness. In full-cue conditions, there is a variety of cues available for perceiving layout (Cutting \& Vishton, 1995) that can influence the path integration performance. Laurent and Cavallo (1985) showed that when subjects have to circumvent several obstacles in order to reach a previously viewed target in full-cue condition, their accuracy is actually better than when they walk directly to it. On the other hand, Philbeck and Loomis (in press) showed that, in the reduced-cue condition used here, the angular elevation of the head was sufficient to correctly encode locational information. In the first experiment, we evaluated how accurate initial perception of the object's orientation was-perception extracted mainly from perspective cues. Observers rotated a model of the previously viewed object until it matched the memorized orientation of its counterpart object.

2. How can we ascertain that observers adopted each processing mode? In order to make sure that observers allocated attention toward the relevant spatial information for each of the processing modes mentioned above, we had them verbalize continuously the output of their "computational imagery" (Glasgow \& Papadias, 1992) while walking on an L-shaped trajectory. Accordingly, observers memorized the initial location and orientation of the object. Then, if asked to focus on the object's perspective (object-centered task, or OC task) they had to state loudly which side of the object could be viewed mainly from their current location (as if they could face the object and see it). In the other task, subjects stated loudly how many steps they walked and any turn taken, which allowed them to deduce the object's perspective at a final vantage point given the trajectory they walked and the initial view of the object (trajectory-centered task, or TC task). Finally, if observers correctly performed each processing mode, differential effects were expected on both the locomotor velocity and response latencies.

In the OC task, subjects mainly rotated the object mentally with respect to their current position while walking without vision. In the TC task, subjects were concerned only with mentally tracing the path they walk, and with refreshing the initial view of the object in memory. Accordingly, during the walk, the TC subjects had to assess the distance they walked and any angle turned. For the OC subjects, this updating of distance and angle had to be accompanied by a continuous updating of both the location and the orientation of the object itself. Therefore, during the walk, the OC task is more demanding than the TC task in terms of processing capacity or cognitive load. One would expect slower body movements for the OC subjects than for the TC subjects as a result. In order to verify this effect, one experimenter led observers to the terminal position, controlling only that they did not deviate from the imposed path. The subjects were explicitly 
encouraged to control the speed of their locomotor movement (i.e., slowing down or speeding up the movements when necessary) so that the task would be performed optimally.

At the end of the walk, observers were asked to face the (nonvisible) object in order to indicate where they thought it was located. Following Haber, Haber, Penningroth, Novak, and Radgowski (1993), who found that pointing methods using body parts (e.g., nose, chest, or index finger) or extensions of body parts (e.g., hand-held cane or short stick) lead to more accurate response than those using dial pointer, drawing, or clockface labels, we chose the facing method as a pointing measure. Our subjects were asked to align their body, head, and line of sight together toward where they thought the object (i.e., its vertical bar) was located. Once the object was faced, observers gave the orientation of the object (i.e., its horizontal bars) by rotating a model of the object inside a box fixed relative to the observer's head to its estimated perspective relative to the current vantage point. Since, in the OC task, observers updated this attribute along with their walk, we expected shorter reaction times here than in the TC task, for which they had to make additional computations to end up with the object's orientation response.

3 . How can we separate the sources of error in the observer's response concerning the object's location from those concerning its orientation? Several factors might be responsible for the errors observers may make when indicating the location or orientation of the object.

First, it is possible that the subjects know where the object is, but are poor at facing it for indicating its location. This would contradict Haber et al. (1993), showing that the facing method is the more accurate for such kind of response. Yet, if this is still the case, one might compare the orientation-of-the-object response given by the subject to the correct response expected from his/her current location, even if the object is not faced correctly. In such a case, we would consider the facing response as being a noise not worth being taken into account.

Second, it is possible that subjects know where the object is, but are poor at indicating the orientation of the object. Therefore, Experiment 1 served as a calibration for testing the ability of the observer to encode and indicate the orientation of the object under a reduced-cue condition (the borders of the object were glowing in darkness). We might then in the walking experiment correct for those errors by subtracting them from the response of the subject.

Third, the observers might have misjudged their selfto-object distance from the initial vantage point and thus the location of the object. This sounds possible given that the object was observed in a reduced-cue condition, but Philbeck and Loomis (in press) showed that in such circumstances, the angular elevation of the head was sufficient to correctly encode locational information. Fukusima, Loomis, and Da Silva (in press) showed that by triangulating at least two facing directions (final headings) obtained from separate terminal positions, it is possible to compute the observer's subjective location of the object. If errors on self-to-object distance occurred, we would expect the subjective location of the object to fall on the observers' initial line of sight (i.e., when encoding the object's location and orientation), rather than perpendicularly to it.

Given the object at a fixed location in space, if the triangulated locations appear inconsistent from one trial to the other, we can propose a fourth source of error-that the observers misjudged the distance they walked. Therefore, from the facing performance, we may try to infer what was the subjective terminal position of the subject on the path he/she walked by shifting along the path the final direction he/she faced (heading) until it crosses the real location of the object. Then, we can compare the orientation-of-the-object response of the observer to the one expected from the subjective vantage position. The path used in the present experiment was L-shaped, and observers were stopped either on the first segment or on the second one. If the errors on the orientation of the object are different for the terminal positions between both segments, this surely means that the turn in the walk is a supplementary source of error.

In summary, our contention is that until now the stages of processing subsequent to path integration have been neglected in the literature on nonvisual navigation. Nevertheless, authors agree that the processing of locational information demands central processing (Böök \& Gärling, 1980; Corlett et al., 1990; Lindberg \& Gärling, 1983). In a preliminary calibration test (Experiment 1 ), we evaluated the ability to memorize the orientation of a 3dimensional object in space. In Experiment 2, we compared the characteristics and effects of two processing modes on the updating of an object's orientation and location during nonvisual navigation, as well as on the response latencies and locomotor activity. We also tested the predictive efficiency of several mental imagery tests and questionnaires on both the memory for object's orientation and its updating performance.

\section{EXPERIMENT 1}

In Experiment 1, we examined how the orientation of a 3-D object (an uppercase letter F) previewed in a reducedcue condition (i.e., the borders of the object were glowing in darkness) is perceived and reported by stationary subjects, using a miniature model of the object (inside a box fixed with respect to observer's head). The orientation of this object was given by the direction in which its two horizontal bars were pointed. The results of this calibration test are used in Experiment 2 to explain the errors on the updating of the object's orientation when the subject is walking without vision.

\section{Method}

\section{Subjects}

Twelve subjects participated in the experiment $(5$ men and 7 women). All were undergraduate students in their early 20 s and. with two exceptions, were paid to participate. All subjects had normal or corrected-to-normal vision and were naive as to the purpose of the experiment. First, each subject was administered the Vandenberg and Kuse (1978) Mental Rotation Test (MRT), Gor- 
don's (1949) Test of Visual Imagery Control (TVIC), and Marks's (1973) Vividness of Visual Imagery Questionnaire (VVIQ). In the MRT, the subjects have to identify two out of four block shapes that are congruent with a standard shape but rotated in space. The TVIC measures the ability to control visual images; subjects are asked to form an image of a car and to "see" it change color, position, or orientation. Subjects indicate on 3-point scale whether they have succeeded in visualizing the altered scene. Finally, the VVIQ is a 16-item questionnaire that requires subjects to form visual images of various scenes, evaluating image vividness on a 7-point scale. It should be noted that many subjects with poor visual imagery (on a self-report scale) have excellent spatial skills as evaluated by other tests (see Poltrock \& Agnoli, 1986, for a review).

\section{Experimental Objects}

Two objects were constructed of wood in the form of the letter $F$. The first one ("large F") was $45 \mathrm{~cm}$ high, and its borders $(2.5 \mathrm{~cm}$ width) were painted with a phosphorescent material to render them visible in complete darkness (Figure 1A). The second object was one fifth the height of the first one, and was mounted inside a clear plastic box that could easily be held by the subject. The orientation of this "miniature F" was controlled by a small rotating knob under the box and indexed by a protractor that rotated with the model (on the top of the box; Figure (B). Photometric measurements made
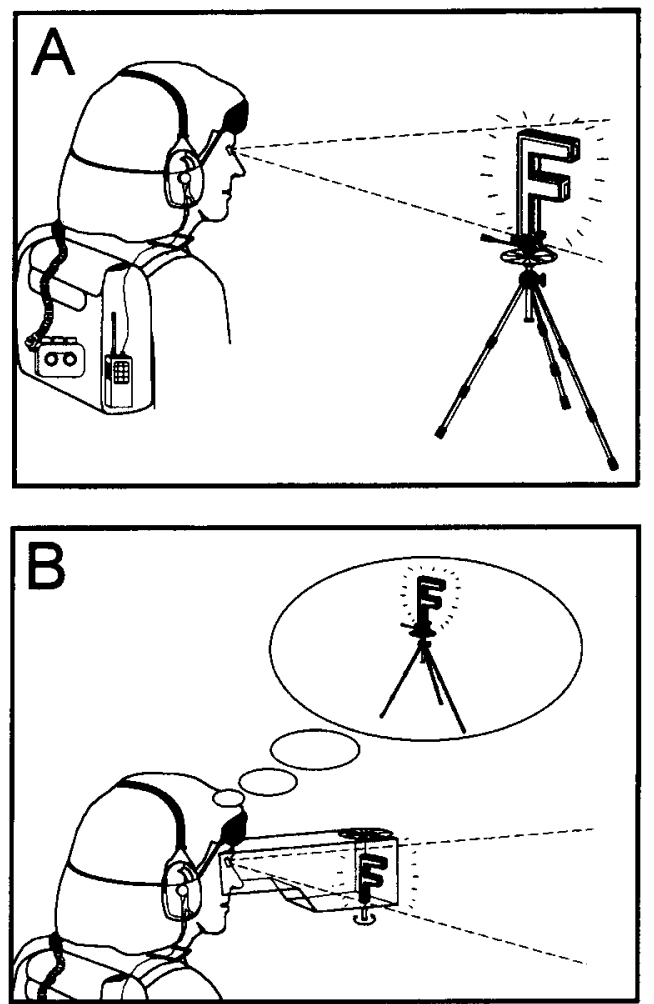

Figure 1. The vertical bar of the 3-D object (an uppercase letter F) defines its location, whereas the direction in which its two horizontal bars were pointed (with respect to both the observer's line of sight and geographic space) defines its orientation. (A) Viewer with headset (blindfold, earphones, white noise tape player, HF receiver) memorizing the target orientation. The viewer, with eyes closed and blindfold on, keeps the target orientation in memory, and after a signal opens the eyes with the blindfold removed. (B) Then the viewer adjusts the model of the object manually to match the remembered perspective. with a Minolta Luminance meter (Model LS-100) indicated that the objects had a luminance $(L)$ of $0.047 \mathrm{~cd} / \mathrm{m}^{2}$ after the room lights were extinguished, which decayed exponentially with a time constant of $43 \mathrm{sec}$. Even for an observation or a response taking up to $30 \mathrm{sec}$ to complete (which never happened), the final luminance of $0.02 \mathrm{~cd} / \mathrm{m}^{2}$ would remain above photopic threshold $\left(0.01 \mathrm{~cd} / \mathrm{m}^{2}\right)$.

\section{Procedure and Experimental Design}

The subjects first listened to the instructions concerning the task, and then were given one practice trial. To exclude auditory cues, subjects wore headphones carrying white noise from a portable tape player. However, instructions from the experimenter were also delivered to their right ear via radio transmission. Each subject was asked to look at and memorize the orientation of the large $F$ at a distance of $1.84 \mathrm{~m}$, the top of the target object being presented $3.5 \mathrm{~cm}$ below eye level (Figure 1A). They were then instructed to close their eyes and wear a blindfold. The object was subsequently covered with black paper. After $15 \mathrm{sec}$, the subject was asked to open his/her eyes and remove the blindfold. The subject then examined the small $\mathrm{F}$ and set it to the remembered orientation of the large F (Figure 1B). This orientation was defined as the direction of the two horizontal segments of the large $F$ in geographic space. Small motions of the head were discouraged, and accuracy was encouraged. After having oriented the small $F$, the subject closed her/his eyes, put on the blindfold, and waited for the next trial.

The subjects performed the preceding calibration task before each condition (OC and TC task) of Experiment 2. Each calibration session was composed of 16 trials: 2 trials each of 8 possible orientations $\left(-157.5^{\circ},-112.5^{\circ},-67.5^{\circ},-22.5^{\circ}, 22.5^{\circ}, 67.5^{\circ}\right.$. $112.5^{\circ}$, and $157.5^{\circ}$ in viewer-centered coordinates) of the object (Figure 2 ). The orientations of the object were presented in a pseudorandom order. Because of time limitation, orientations falling on the viewer's line of sight $\left(0^{\circ} / 180^{\circ}\right)$ or perpendicularly to it $\left(-90^{\circ} / 90^{\circ}\right)$ were not presented. The starting orientation of the hand-held object model was also chosen pseudorandomly and independently. Each experimental session lasted about $30 \mathrm{~min}$. In summary, the experimental design consisted of two completely crossed within-subjects factors, the task factor (with two levels: the $\mathrm{OC}$ or TC conditions of Experiment 2) and the object orientation factor (with eight levels), with 12 subjects and two repetitions per cell.

\section{Results and Discussion}

\section{Evidence for a Perceptual Bias Toward Diagonal Orientations}

Figure 2 shows the means and 1 standard error of the mean for each object orientation in a polar representation. Averages and dispersions were calculated over 12 values (one mean per subject per object orientation). Circle A shows the results of this control test prior to the OC task session, and Circle B shows those for the TC task session. Errors (difference between correct response and the viewer's estimation) were coded so that negative errors indicate an underestimation and positive errors an overestimation with respect to the correct object orientation and observer's position $\left(0^{\circ}\right)$. Under- or overestimations refer to object orientations chosen by the subject that are rotated toward the viewer's location or the opposite direction, respectively, for each targeted orientation. Subjects aligned the small $F$ quite accurately with respect to the actual orientation of the large $F$. The average error was -0.8 for the trials that preceded the OC task in Experi- 


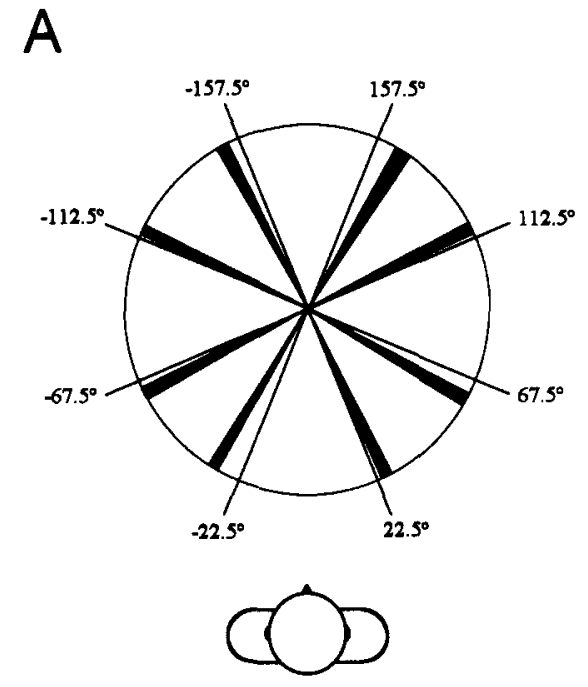

B

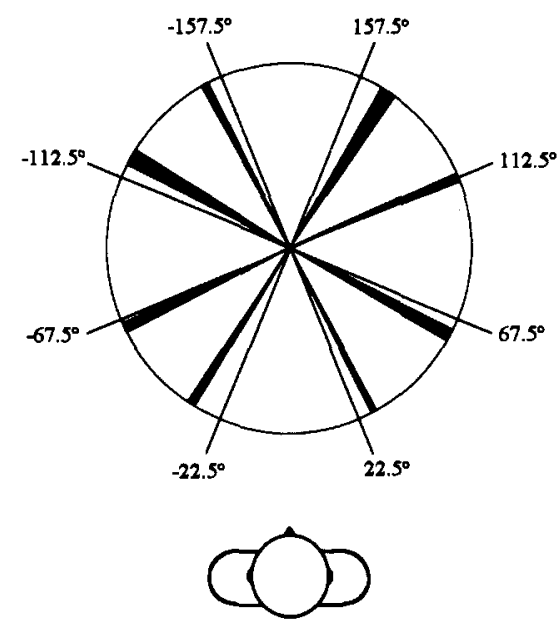

Figure 2. Polar representation of the results of Experiment 1 as a function of each target orientation and Experiment 2 task. Each circular sector is centered on the mean adjustment response. Shaded areas represent \pm 1 standard error of the mean. The respective correct object orientation is indicated by the prolonged lines next to each sector. Circle A shows results for the object-centered session, and circle $B$ for the trajectory-centered session.

ment 2 , and mean individual errors ranged from -21.8 through 20 across the different object orientations. For the calibration trials preceding the TC task, the average error was -0.2 and ranged from -24.5 through 20.3 across the different object orientations. An analysis of variance (ANOVA) of the signed errors showed a significant $[F(7,70)=20.58, p<.0001]$ effect of object orientation only, whereby estimates tend to be "attracted" by diagonal $\left(-45^{\circ} / 135^{\circ}\right.$ and $\left.45^{\circ} /-135^{\circ}\right)$ orientations of the object (Figure 2). Interestingly, estimations of object's orientation are consistent all around the object axis of rotation; for example, the overestimation for the -22.5 direction is aligned with the underestimation of the 157.5 direction. The errors we observed did not reflect classical dis- tortions such as depth foreshortening (Toye, 1986; Wagner, 1985), which would bias the response toward the plane perpendicular to the viewer's line of sight (i.e., $-90^{\circ} / 90^{\circ}$ ). Instead, the perceptual bias toward the diagonal orientation may reflect an attempt to facilitate the encoding of the object's orientation. The object's diagonal orientations would be categorized into one direction intermediate between viewer's line of sight and its perpendicular plane. Finally, we found a significant correlation $(r=-.63, p<.03)$ between individual scores on the TVIC and individual mean absolute error in the present calibration task.

\section{EXPERIMENT 2}

In Experiment 2, each subject first memorized the location and orientation of the object from an initial viewing position. Then the observer was led blindfolded by the experimenter to one of six possible terminal positions along an L-shaped trajectory. During the walk, the experimenter placed his hands on the observer's shoulders in order for the subject not to deviate from the imposed path only. The subject was encouraged to control her/his walking speed in order to solve the task optimally. The large $F$ was hidden, and the subject was then asked to face the location of the object. After removing the blindfold, the subject used the miniature model to indicate the orientation of the object relative to the current position of the observer. In the OC task, subjects were instructed to concentrate on the object orientation while walking, whereas in the TC task, they were asked to concentrate on the trajectory they walked. We expected an effect of task on the locomotor velocity for both linear and angular movements, and on reaction times for the object's orientation response, as stated in the Introduction. Errors on the localization and orientation of the object were also considered. In addition, we expected shorter errors for object orientations pointing to the observer's line of sight or perpendicularly rather than for other directions because of easier encoding (although we didn't examine performance for these orientations in Experiment 1).

\section{Method}

Subjects

The subjects were the same as those in Experiment 1 .

\section{Experimental Space and Objects}

We used the same stimuli and headset as in Experiment 1. In addition, the 3-D spatial position of the head of the subject was recorded and computed by an ELITE, an infrared video motion analyzer (Ferrigno \& Pedotti, 1985). Subjects wore helmets bearing two antenna-like bars aligned with their line of sight, with an infrared-reflective marker at their tips. The two cameras recorded marker locations (Figure 3 ) at a rate of 100 images per second each; these were separated by $4.5 \mathrm{~m}$ and mounted at $3 \mathrm{~m}$ above ground level.

\section{Procedure and Experimental Design}

Sequential description of the task. The subject was given the task instructions outside of the experiment room. One experimenter (El) monitored the ELITE system and instructed the subject through a wireless system. The blindfolded subject was guided 


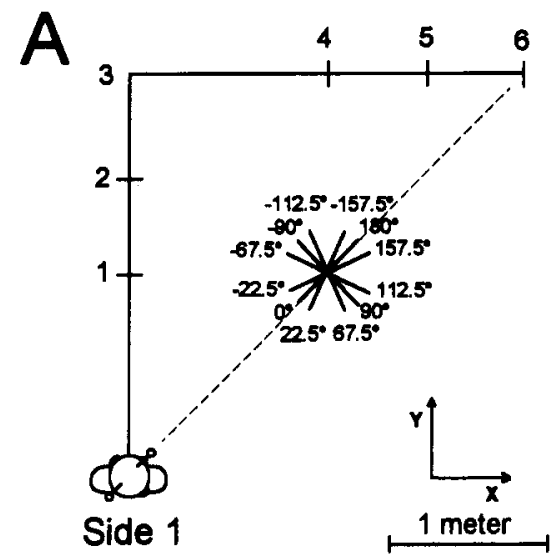

$\checkmark:$ Camera 1

Camera 2: 0

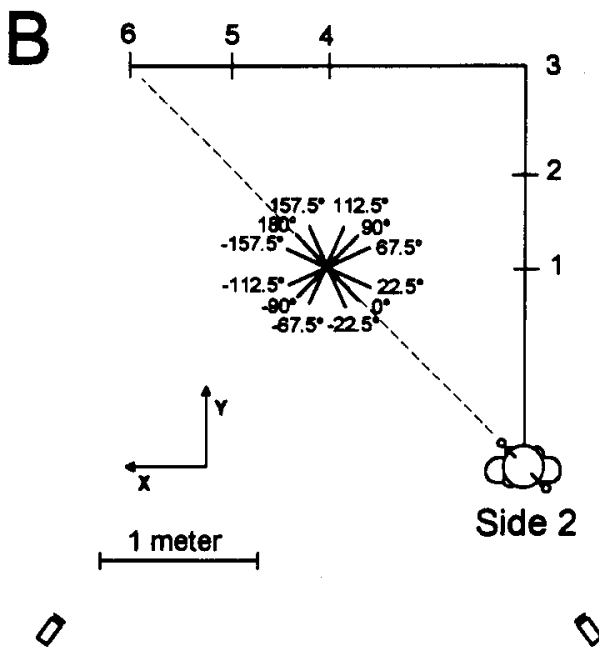

Figure 3. Experiment 2: Map view of the arrival points (1 to 6) for both departure sides (panels $A$ and $B$, respectively). The observer is memorizing one of the 12 possible target orientations expressed in viewer-centered coordinates, and with respect to observer's line of sight (dashed line) facing the vertical bar of the object.

to the experiment room by a second experimenter (E2) and was led to a chair. The subject opened her/his eyes and faced a uniformly white, very bright surface (luminance $=115 \mathrm{~cd} / \mathrm{m}^{2}$ ) fixed on the wall, which maintained the eyes in a state of light adaptation. This prevented the observer from perceiving very dim cues in the room, which was darkened when the subject was encoding the orientation of the luminous object. When the room lights were turned off, the subject closed her/his eyes, put the blindfold on, stood up, and was led by E2 (using a flashlight) to an initial position facing the turn of the L-shaped trajectory (Figure 3 ). The subject removed the blindfold, turned his/her head toward the object location, and memorized its orientation. The object was oriented toward 1 of the 12 different viewer-centered directions illustrated in Figure 3 and numbered in viewer-centered coordinates. Once the object orientation was memorized, the subject closed her/his eyes, turned his/her head back perpendicular to her/his chest, indicated when ready, and was then guided (by hands on both shoulders from behind) along a path to one of 6 possible terminal positions along the
L-shaped path. (The distances from initial position were as follows: $1=1.30 \mathrm{~m} ; 2=1.95 \mathrm{~m} ; 3=2.60 \mathrm{~m} ; 4=3.90 \mathrm{~m} ; 5=4.55$ $\mathrm{m}$; and $6=5.20 \mathrm{~m}$ ). There were two mirror trajectories, and thus two possible departure sides (Figure 3 ). As soon as the terminal position was reached, the ELITE recording began. The subject was instructed to turn toward the object location (as defined by its vertical bar), keeping the head and body aligned together with the line of sight, and to imagine its orientation from this new vantage point. The subject indicated when she/he got a clear mental image of the object orientation, and the ELITE was stopped. The subject then opened her/his eyes, removed the blindfold, and adjusted the "small F" so that it matched her/his mental representation of the object. The subject was then blindfolded and guided back to the chair and remained there until the next trial.

Cognitive task. The cognitive task differed across experimental sessions. In the OC task, the subject focused attention on the location and orientation of the imagined object during the walk. Following a given code (one number per object side), the subject indicated loudly, during the walk, which of the four sides (front, right, back, or left) of the pointing object would be "seen" mainly from her/his current position. In the TC task, the subject was instructed to pay attention to her/his path, counting out loud the number of steps and telling the direction (either left or right) of any turns taken. From the terminal position, the subject was asked to reconstruct the visual perspective of the hidden object by recalling its initial view from the starting position and taking into account the walked trajectory. Subjects were allowed only one practice trial before the experiment started, and were asked to walk at self-judged optimal speed in order to perform the tasks.

Figure 4 shows the main experimental events as well as the time measurements we performed. The duration of the walk from the initial to the terminal position was measured with a chronometer clock to an accuracy of $10 \mathrm{msec}$. Object localization time (i.e., head turn latency + head turn duration toward the object) and object orientation latency were measured from the ELITE (head movement) recordings.

Subjects were pseudorandomly assigned to two groups that differed according to the task session order: For 6 subjects, the $O C$ task was first and the TC task was second (OC-TC order); for the other 6 , the order was inverted (TC-OC order). This was done to check for training effects. Each group underwent two experimental sessions, the OC and TC tasks ("processing mode" factor). Each experimental session had 24 trials (not including the practice trial): two repetitions of six terminal positions by two departure sides. The 12 viewer-centered object orientations for each combination of terminal position by departure side were pseudorandomly counterbalanced across all the subjects in an incomplete balanced design (Cochran \& Cox, 1957) - that is, nine object orientations per subject with two repetitions of three of them. Each subject kept the same set of "object orientation by terminal position by departure side" combination for both tasks. For statistical analytic purposes, we will consider a two-level object orientation factor: those falling on the line of sight together with those perpendicular to it (respectively, $0^{\circ} / 180^{\circ}$ and $-90^{\circ} / 90^{\circ}$ in viewer-centered coordinates) versus all of the other orientations (Figure 3 ). To summarize, we used the following experimental design: one between-subjects factor (two orders of session) and four completely crossed withinsubjects factors (object orientation factor with two levels, processing mode factor with two levels, terminal position factor with six levels, and departure side factor with two levels) with 6 subjects and two repetitions per cell.

\section{Results and Discussion}

\section{Locomotor Activity and Processing Times}

A glance at Figure 4 is recommended in order to refresh the reader's memory on the experimental events related 


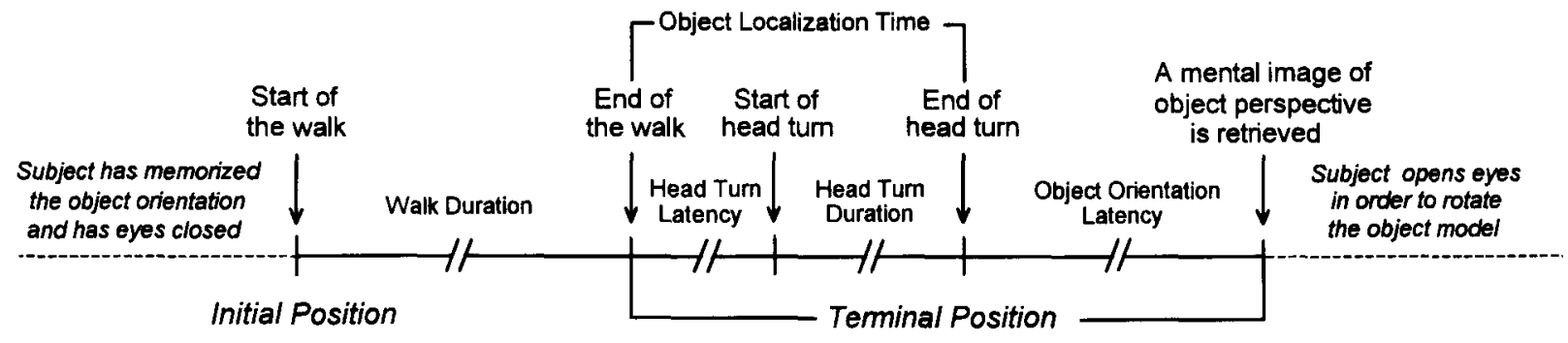

\section{Time flow}

Figure 4. Experimental events flowchart for Experiment 2.

to each of the following dependent variables. All the time measurements are in seconds.

Walk duration. Figure $5 \mathrm{~A}$ shows the results of the $\mathrm{OC}$ and TC tasks as a function of each level of the terminal position factor. An ANOVA of walk duration showed that subjects walked significantly faster $[F(1,10)=21.68$, $p=.0009]$ in the TC task than in the OC task. There was also an obvious effect of terminal position (walked distance) on walk duration $[F(5,50)=1,037.44, p<.0001]$ and a significant interaction between processing mode and terminal position $[F(5,50)=6.89, p=.0001]$, reflecting the fact that subjects turned more slowly at the corner (Terminal Position 3 ) in the OC task than in the TC task. This led to significantly $[F(1,50)=32, p<.0001]$ larger duration differences between $\mathrm{OC}$ and TC tasks for Terminal Positions 4, 5, and 6 than for Terminal Positions 1,2 , and 3 . No effect of the other main factors was found.

Head turn latency. This time measurement started at the end of the walk and stopped at the initiation of the head turn toward the object. An ANOVA of head turn latency showed no significant $[F(1,10)=0.93$, n.s. $]$ difference between head turn latency for OC $(M=1.19$; $S D=$ $1.02)$ and TC $(M=1.39 ; S D=1.31)$ tasks. The ANOVA showed a significantly $[F(1,10)=8.64, p=.015]$ shorter head turn latency for Repetition $2(M=1.15 ; S D=1.09)$ than for Repetition $1(M=1.43 ; S D=1.24)$ and no effect of the other main factors.

Head turn duration. This time measurement is the duration of the head movement toward the object. The ANOVA of head turn duration showed a significant $[F(1,10)=5.10, p=.048]$ effect of (OC-TC vs. TC-OC) session order. In order to disentangle the learning that occurred from one session (or processing mode) to the other from the effect of processing mode on head turn duration, we plotted the results for the tasks done first only (Figure 5B). Indeed, there was a significantly $[F(1,10)=$ $17.32, p=.002]$ shorter head turn duration for TC task in the TC-OC order than in the OC task in the OC-TC order (Table 1), and no significant $[F(1,10)=3.37$, $p=.1$ ] difference between both processing modes when they were done second. These results may indicate that when each processing mode is used for the first time, there is a need to turn the head slowly toward the object location in the $\mathrm{OC}$ task in order to update its perspective carefully. Alternatively, the TC subjects concentrated on their walk. Since they cannot generate their response until they are in the terminal position, the head rotation is not as important as for the OC subjects, who concentrated on the relation between their head and the stimulus throughout the task. In short, the difference is not due just to what OC subjects were doing, but also to what the TC subjects did. This finding is consistent with the interaction between processing mode and terminal position on walk duration that we described above, indicating slower movements for OC subjects at the turn during the walk than for TC subjects.

Finally, the ANOVA showed a significantly $[F(1,10)=$ $10.28, p=.009]$ shorter head turn duration for Repetition $2(M=2.87 ; S D=1.40)$ than for Repetition $1(M=$ $3.14 ; S D=1.79$ ) and no effect of the other main factors.

Object localization time. This processing time is obtained by summing both the head turn latency and the head turn duration. There was no significant difference between both tasks $[F(1,10)=0.29, p>.10]$ on the global

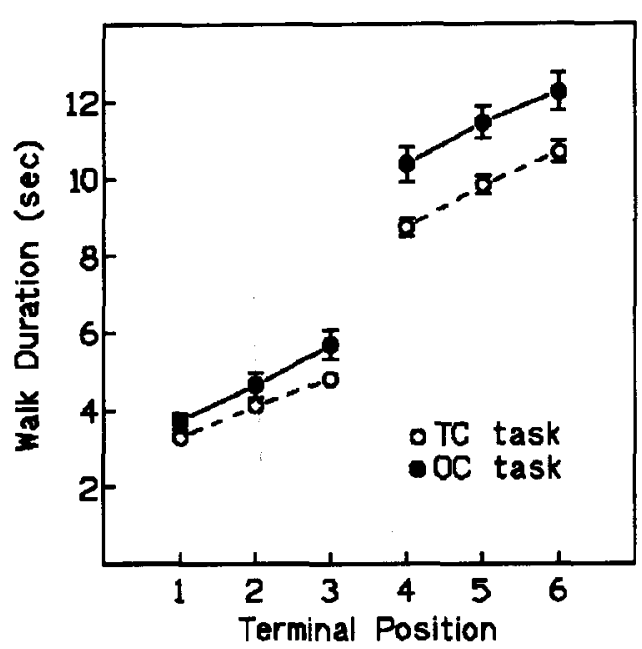

Figure 5. Mean walk duration results and their standard error bars plotted as a function of each level of terminal position for object-centered (OC) and trajectory-centered (TC) tasks. 
Table 1

Mean Head Turn Duration (in Seconds) and Standard Deviations for Both $O C$ and TC Tasks as a Function of Session Order

\begin{tabular}{cccccccc}
\hline & \multicolumn{2}{c}{ OC-TC Order } & & \multicolumn{2}{c}{ TC-OC Order } & & \\
\cline { 2 - 3 } Task & $M$ & $S D$ & & $M$ & $S D$ & & $S$ \\
\hline OC & 3.99 & 2.12 & & 2.46 & 0.94 & 3.22 & 1.81 \\
TC & 3.15 & 1.58 & & 2.42 & 0.96 & 2.79 & 1.36 \\
& 3.57 & 1.92 & 2.44 & 0.95 & & \\
\hline
\end{tabular}

object localization time. The ANOVA showed a significantly $[F(1,10)=11.54, p=.007]$ shorter object localization time for Repetition $2(M=4.01 ; S D=2.10)$ than for Repetition $1(M=4.57 ; S D=2.55)$ only, and no effect of the other main factors.

Object orientation latency. Object orientation latency (Figure 6) started once the observer faced the object and stopped when she/he said that a mental image of its perspective was retrieved. An ANOVA of this latency showed a significant $[F(1,10)=17.90, p=.0017]$ effect of the processing mode; specifically, latencies for the OC task $(M=3.59 \mathrm{sec} ; S D=1.97 \mathrm{sec})$ were shorter than those for the TC task $(M=7.84 \mathrm{sec} ; S D=5.57 \mathrm{sec})$. Using Bayesian procedures, we evaluated what would be the minimum effect $(\delta)$ of processing mode expected in the parent population (Bernard, 1994), according to our data. With Bayesian ANOVA techniques (Rouanet \& Lecoutre, 1983 ), it is possible to build a distribution over the parent effect $\delta(\delta=\mu 1-\mu 2)$ from the experimental data. The distribution is centered on the observed effect $d(d=$ $m 1-m 2)$, and its dispersion [i.e., $(d / \sqrt{F})^{2}$, or the observed effect $d$ divided by the square root of its corresponding ANOVA $F$ ratio value, at the power of two] translates the potential of generalizability over $\delta$ that is carried by the experimental information. The magnitude

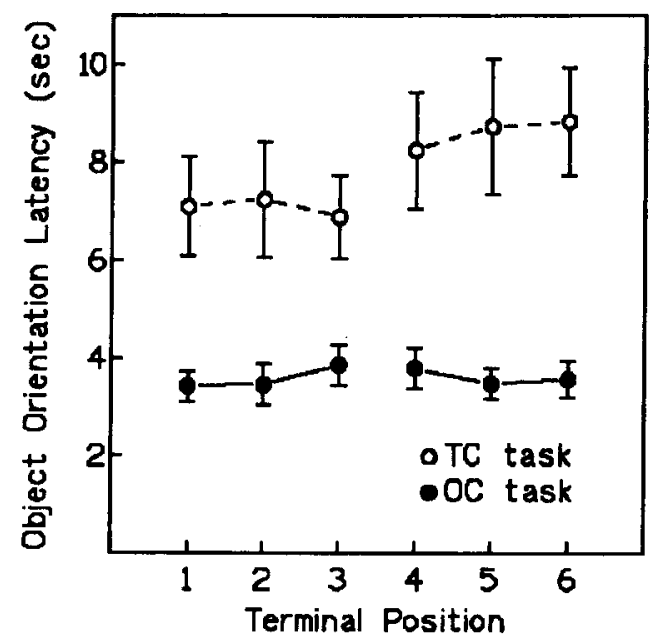

Figure 6. Mean results for the object orientation latency (see also Figure 4) and their standard error hars plotted as a function of each level of terminal position for object-centered (OC) and trajectory-centered (TC) tasks. of the effect can be assessed through credibility limits obtained from this distribution (Bernard, 1994; Rouanet, 1996). Descriptively, here, the observed processing mode effect on object orientation latency is $4.25 \mathrm{sec}\left(M_{\mathrm{TC} \text { task }}\right.$ $\left.M_{\mathrm{OCtask}}\right)$. Its corresponding theoretical distribution is $t 10\left(4.25,1.00^{2}\right)$. From the $t$ distribution tables, we find with a Bayes-fiducial probability of $95 \%$ that $\delta$ (the mean additional processing time in the TC task, as compared with the $O C$ task) is at least $+2.43 \mathrm{sec}$.

The ANOVA showed no effect of terminal position on this latency $[F(5,50)=1.76$, n.s. $]$. However, there was a significant $[F(1,50)=8.14, p=.006]$ interaction between processing mode and Terminal Positions 1, 2, and 3 together against 4,5 , and 6 . This interaction is probably a consequence of turning the corner in the path from Terminal Position 3 to 4, which causes, in the final "spatial inference" computations of TC task, one more rotation to be taken into account.

Object localization and orientation errors. The following analyses of object localization and orientation errors were conducted under the two general assumptions that observers correctly perceived (1) their course (the direction of their travel) while walking blindfolded, and (2) the right angle turn between the two path segments (the one containing Terminal Positions 1,2, and 3 and the other containing Terminal Positions 4, 5, and 6). The last assumption is largely supported by previous studies showing that navigation and orientation judgments tend to be carried out in (and distorted toward) a normalized rightangle grid (Sadalla \& Montello, 1989; Stevens \& Coupe, 1978) and also by postexperimental interview. It is also supported by Fujita, Klatzky, Loomis, and Golledge's (1993) encoding-error data, obtained from subjects who walked back to their initial location after a guided walk without vision on the two first segments of a triangular path.

\section{Hypotheses on Main Source of Error}

1. Estimation of final heading. Heading error $(\theta$ error) was computed (Figure 7A) by subtracting the desired correct final heading (facing direction) toward the object location in the room to subject's actual heading. Figure $7 \mathrm{~B}$ reports mean heading $(\theta)$ error as a function of each terminal position level for OC and TC tasks. Negative and positive errors indicate, respectively, that observers either undershot or overshot the desired angle to face the object. An ANOVA showed that $\theta$ error increased significantly $[F(5,50)=10.98, p<.0001]$ and similarly with Terminal Positions 1,2 , and 3 and 4,5 , and 6 . There was no effect of the other main factors on $\theta$ error.

Assuming that $\theta$ error reflects an error in head turn execution, instead of an error on the updating of object location, it is possible to analyze object orientation error (OOE) by subtracting the correct response $\Phi$ expected at the actual terminal position from the subject's response $\phi$ (Figure 7A). Accordingly, positive values refer to overrotations of the small $\mathrm{F}$ (clockwise $[\mathrm{CW}$ ] for Side 2, counterclockwise $[\mathrm{CCW}]$ for Side 1), whereas negative 

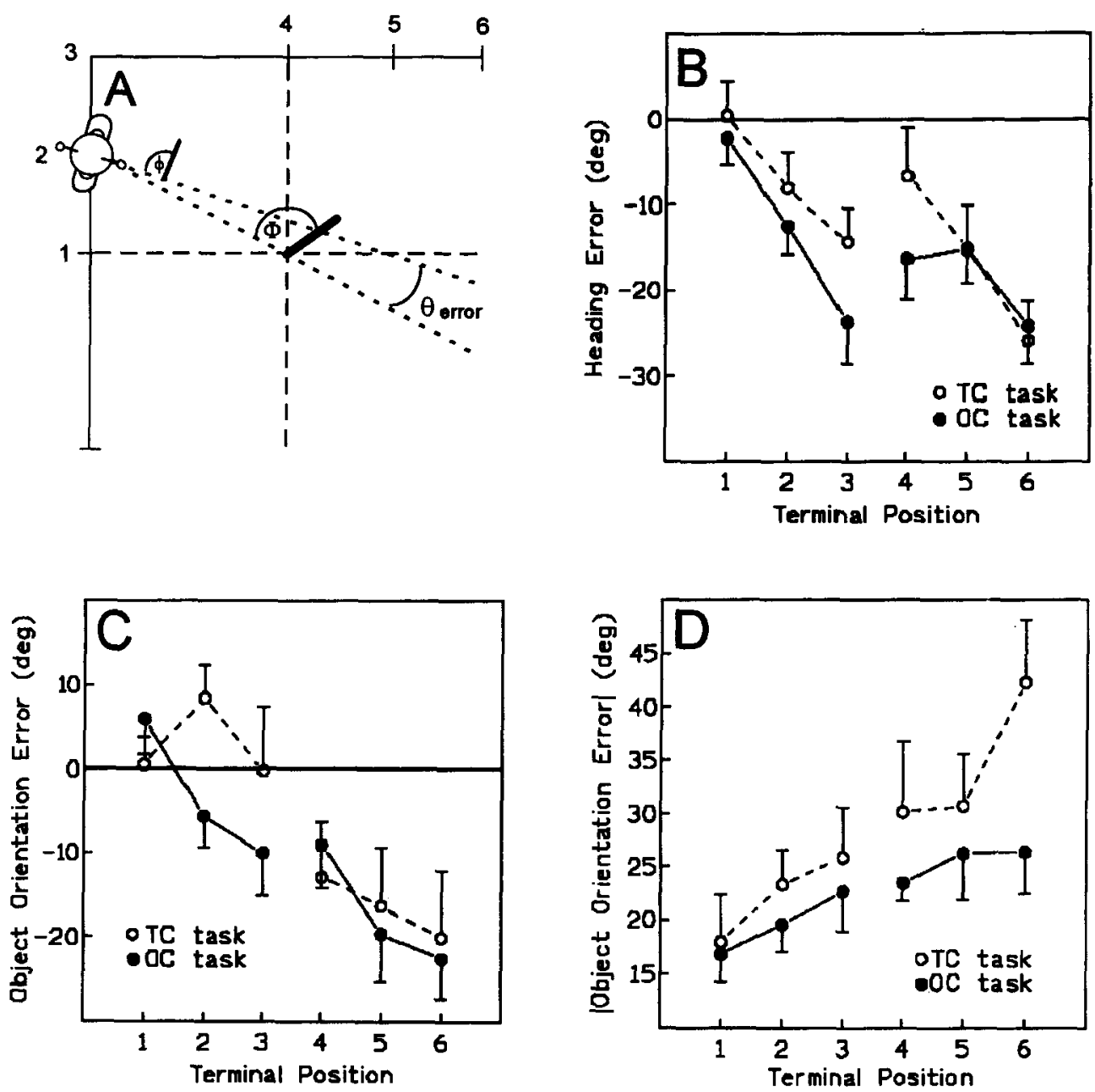

Figure 7. A: Map view of the final heading error ( $\theta$ error) for a subject stopped at Terminal Position 2 (for Side 1). Short thick lines represent the orientation of both the small and the large $F$, whose vertical bars should fall onto line of sight. One dotted line shows observer's actual facing direction, whereas the other shows the correct response. $\phi$ is observer's response for the orientation of the object, and $\Phi$ is the correct response from the current vantage point. Object orientation error $(O O E)$ is given by $\phi-\Phi$, Mean results for $\mathrm{B}$, heading error ( $\theta$ error); $\mathrm{C}, \mathrm{OOE}$; and $\mathrm{D},|\mathrm{OOE}|$ (OOE mean absolute error), and their standard error bars are plotted as a function of each level of terminal position for the object-centered $(\mathrm{OC})$ and trajectorycentered (TC) tasks.

values refer to underrotations; that is, the small $\mathrm{F}$ is rotated less than expected (CW for Side 1, CCW for Side 2). OOEs are thus composed between $-180^{\circ}$ and $180^{\circ}$. Figure $7 \mathrm{C}$ shows the mean results and 1 standard error of the mean for the OOE as a function of each level of terminal position for OC and TC tasks. There was a significant $[F(5,50)=8.13, p<.0001]$ effect of the terminal position factor on OOE only, and no effect of the other main factors. OOE is clearly related to the terminal position. The incremental mean angular error per terminal position mean unit $(0.87 \mathrm{~m})$ is $-5.35^{\circ}$, that is, $-6.75^{\circ}$ per walked meter. We do not show any trend analysis on this error since the self-to-object distance was not constant during the walk.

In order to correct OOE data for a possible initial misperception of the object's orientation, we subtracted the adjustment errors obtained in Experiment 1 from the OOE data for each subject and her/his corresponding object orientation response. As a consequence, data for orientations falling on the observer's initial line of sight or its perpendicular plane are not modified since there was no calibration value for these orientations. An ANOVA on those corrected OOE data show no main difference in the results with respect to the initial OOE data. Therefore, errors on initial misperception of the object's orientation do not account for the distribution of OOE errors observed at the different terminal positions.

An ANOVA of $|\mathrm{OOE}|$, that is, absolute errors on object orientation (Figure 7D) showed a significantly $[F(1,10)=$ $6.71, p=.03]$ higher mean error for the $\mathrm{TC}\left(M=28.4^{\circ}\right.$; $\left.S D=27.6^{\circ}\right)$ than for the OC $\left(M=22.5^{\circ} ; S D=18.5^{\circ}\right)$ task because of the presence of $-90,90$, and 180.errors. 
These "mirror image reversal" errors typically occur when mental representations of maps are manipulated (Rossano $\&$ Warren, 1989). The ANOVA also showed a significant $[F(5,50)=4.53, p=.002]$ effect of terminal position on $|\mathrm{OOE}|$. We found no effect of the other main factors on |OOE .

However, the observed underestimation error of the object's orientation increasing with walked distance is rather surprising in view of Pinker and Finke's (1980) finding that mental images rotating in depth (around the vertical gravity axis) "move" a constant fraction farther than the amount defined by the physical rotation of the counterpart object. Therefore, there was an error either in the initial perception of object location (Hypothesis 2 ) or in the estimation of distance walked (Hypothesis 3 ).

2. Estimation of object's location. If there is no error in the final heading toward the object at the terminal position, the initially perceived location of the object can be computed by triangulating mean headings $(\theta)$ obtained at separate terminal positions but equivalent in terms of correct final head turn angle toward the object (i.e., 90 for Terminal Positions 1 and $3 ; 116.565$ for $2-4$; and 135 for 3-6) for each path segment. Figure 8A illustrates such a crossing point resulting from the triangulation of mean $\theta$ obtained at Terminal Position 2 with the mean $\theta$ obtained at Terminal Position 5. Similar crossing points were also computed by triangulating mean $\theta$ at Terminal Positions 1-4 and 3-6 for each task condition.

We examined deviations of the subjective location of the object in both the $x$ - and $y$-axes of the room as compared with the actual location of the object within an equivalent self-to-object coordinate system for both departure sides, centered on the object location $(0,0$ coordinates) and with an initial viewing position at equivalent coordinates $(-1.3 \mathrm{~m}$ on the $x$-axis, and $-1.3 \mathrm{~m}$ on the $y$ axis) for both sides (see also Figure 3 ). In Figure $8 \mathrm{~B}$, the error bars denote \pm 1 standard error of the mean $x$ and $y$ errors represented as a function of task condition for the crossing points derived from mean $\theta$ at Terminal Positions $1-4,2-5$, and $3-6$. Heading triangulations clearly led to inconsistent results with respect to the initial perception of the object's location (Figure 8B). Accordingly, an ANOVA of $x$ and $y$ errors showed a significant $[F(2,20)=$ $61.03, p<.0001]$ effect of terminal-position-derived $\theta$ crossing points on $x$ error only, and no effect of the other main factors. If the errors were due to an initial misperception of self-to-object distance (Philbeck \& Loomis, in press), the different subjective object locations should have fallen on the viewer's line of sight (diagonal line in Figure $8 \mathrm{~B}$ ) during encoding of the initial attributes of the object. This is more or less the case for the 1-4 and 2-5 crossing points but not at all for the 3-6 crossing points, which show a lateral deviation with respect to the viewer's initial line of sight.

Otherwise, we can triangulate those crossing points obtained separately from each departure side within a same room coordinate system. Given the physical object in the center $(0,0$ coordinates $)$ of the room, the initial viewing position coordinates would be $x=-1.3 \mathrm{~m}$ and $y=-1.3 \mathrm{~m}$
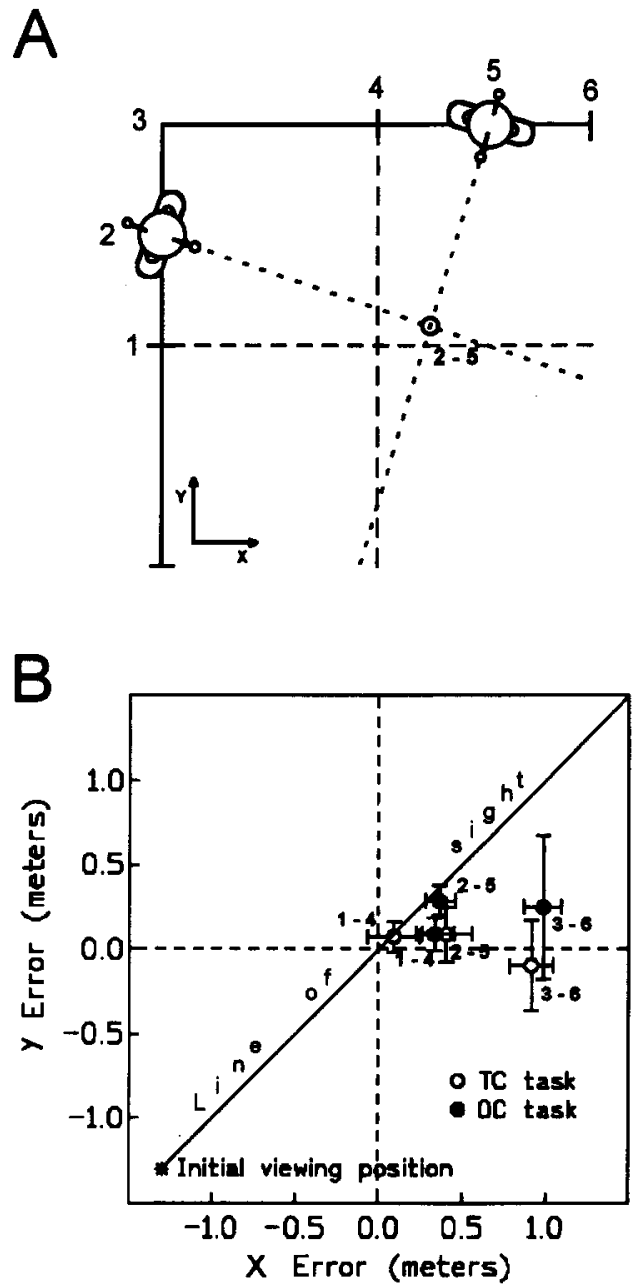

Figure 8. A: Example of a crossing point, indicating the subjective location of the object, obtained by triangulating the headings $(\theta)$ from Terminal Positions 2 and 5. B: Mean $x$ and $y$ errors for each task condition for the crossing points are derived from mean $\theta$ at Terminal Positions 1-4, 2-5, and 3-6. Error bars denote \pm 1 standard error of the mean. The object is located at 0,0 and the initial viewing position at $-1.3,-1.3$, whichever is the departure side.

for Departure Side 1 and $x=1.3 \mathrm{~m}$ and $y=-1.3 \mathrm{~m}$ for Departure Side 2. In that case, the coordinates of the perceived object's location for both departure sides are significantly $[F(1,10)=25.37, p=.0005]$ different on the $x$-axis $\left(M_{\text {side } 1}=0.53 \mathrm{~m}\right.$ vs. $\left.M_{\text {side } 2}=-0.50 \mathrm{~m}\right)$ but not $[F(1,10)=0.001$, n.s. $]$ on the $y$-axis $\left(M_{\text {sidel }}=0.11 \mathrm{~m} \mathrm{vs}\right.$. $M_{\text {side2 }}=-0.12 \mathrm{~m}$ ). The observer's performance, as analyzed in such a room coordinate system, suggests a complete distortion of the object-represented location within the room. If true, this result would be inconsistent with previous research (Fukusima et al., in press; Loomis, Da Silva, Fujita, \& Fukusima, 1992), which, using the method of triangulation by facing a previously seen location, did not show such a systematic locational error. Consequently, since the various terminal-position-derived $\theta$ crossing points are directly related to the walked distance, it is nec- 
essary to reevaluate the data under the assumption that subjects made errors on the perception of the distance they walked, rather than on the initial perception of the object's location.

3. Estimation of walked distance. Under the assumption that the observer has correctly perceived the object location from the initial viewing position and made no final heading errors, $\theta$ error would reflect an error on the estimation of the walked distance. We shifted the observer's actual heading along the path until it crossed the physical position of the object (Figure 9A). This allowed a computation of the observer's subjective position along the path. The difference between the imagined terminal position and the actual terminal position on the walked path defines the walked distance error (WDE). The fact that WDE is derived from $\theta$ is reflected by a similar dis- tribution (pattern) of the results displayed in Figures 7B and 9B. However, since the correct heading angle $\theta$ varies with the terminal position, two identical $\theta$ errors will not give identical WDE values for two different terminal positions along the same path segment. This is the reason that the pattern of $\theta$ errors does not match completely the pattern of WDE. We found a significant $[F(5,50)=2.70$, $p=.03$ ] effect of terminal position on WDE, but no effect of the other main factors.

Furthermore, under the assumption that the observer misperceived the distance walked but correctly perceived the object's location from the initial viewing position, and correctly performed her/his intended head turn toward the object, an analysis was conducted on the difference (the subjective orientation error, SOE) between the actual and expected object orientation response from the
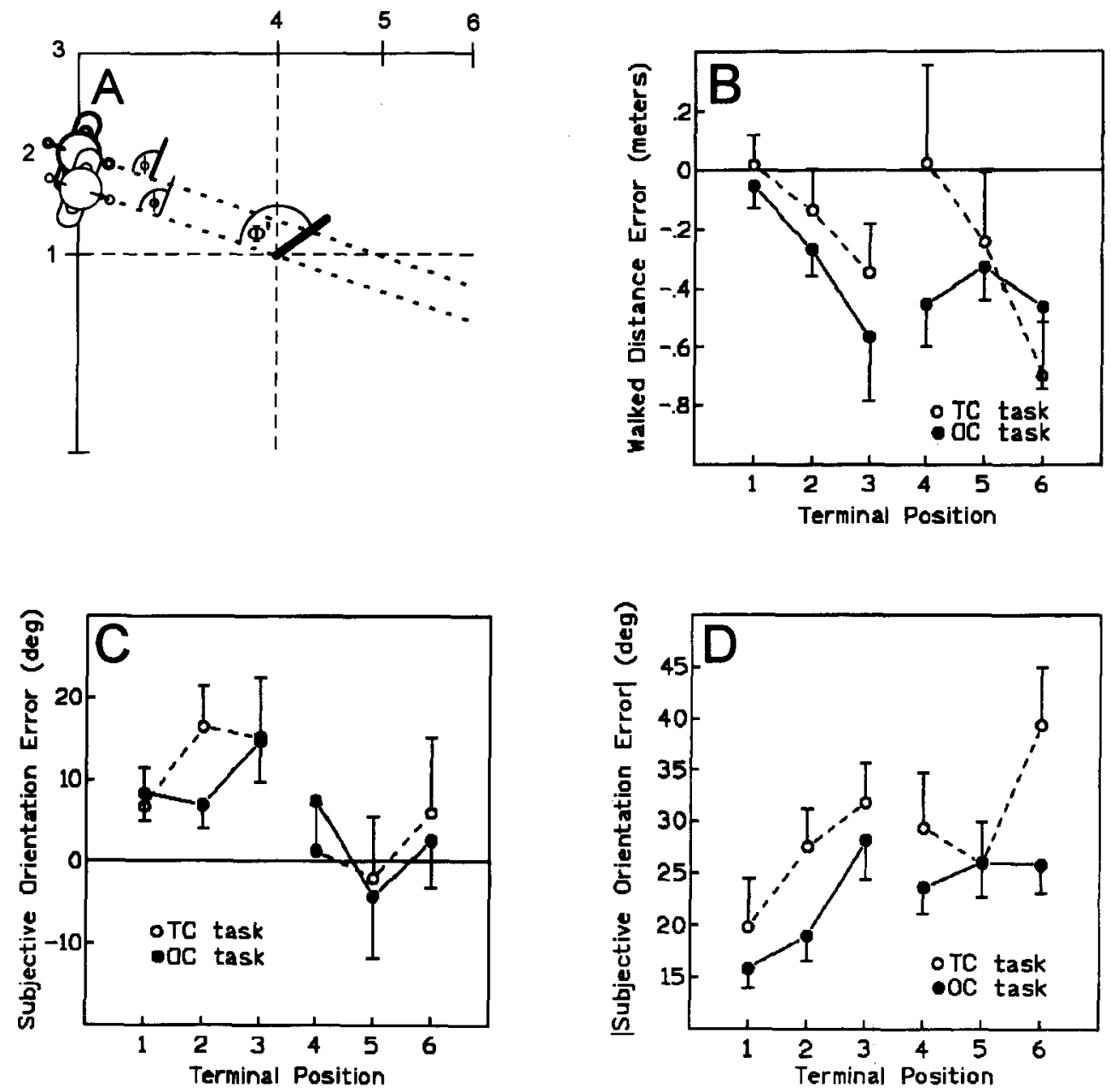

Figure 9. A: Map view of the final heading for a subject (drawn with thick lines) stopped at Terminal Position 2 (for Side 1). By translating the final heading of the observer along the path segment currently walked, until the subject faces the object location correctly, the observer's (drawn in thin lines) subjective location along the path segment is determined. The difference between actual and subjective terminal positions defines the walked distance error (WDE). $\phi$ is subject's response on the object orientation and $\Phi^{\prime}$ is the correct response from the current vantage point. Subjective orientation error (SOE) is given by $\phi-$ $\Phi^{\prime}$. Mean results for $\mathrm{B}$, WDE; $C, S O E$; and $D,|S O E|$ (SOE mean absolute error), and their standard error bars are plotted as a function of each level of terminal position for object-centered (OC) and trajectorycentered (TC) tasks. 
observer's subjective position (Figure 9C). The ANOVA of SOE showed a significant $[F(5,50)=2.83, p=.025]$ effect of terminal position, but no effect of the other main factors. More precisely (Figure 9C), there is a significant $[F(1,50)=9.67, p=.003]$ farther rotation of the object (CCW for Side 1, and CW for Side 2) for Terminal Positions 1, 2, and 3 as compared with correct responses at Terminal Positions 4,5 , and 6 , possibly because of the turn between the two path segments.

We also found that initial orientations of the object falling onto the viewer's line of sight $\left(0^{\circ} / 180^{\circ}\right)$ and perpendicularly to it $\left(-90^{\circ} / 90^{\circ}\right)$ led to significantly $[F(1,10)=$ $13.61, p=.004]$ smaller $\left(M=1.9^{\circ} ; S D=31^{\circ}\right)$ subsequent $\mathrm{SOE}$ as compared with the other initial object orientations $\left(M=8.9^{\circ} ; S D=35.3^{\circ}\right)$. This suggests that the former orientations of the object are less prone to "drift" in memory arising from the kind of tasks and trajectories subjects must undertake, which in turn reduces the error at the subjective terminal position. An ANOVA of $|\mathrm{SOE}|$ (Figure 9D) showed a significant $[F(5,50)=4.36, p=$ $.002]$ effect of terminal position on $|\mathrm{SOE}|$ only.

In summary, under the current hypothesis, errors on walked distance account better for the estimated orientation of the object from the current terminal position, assuming also that the turn between the two legs of the Lshaped path was an additional source of error. However, we still cannot assess the contribution of the initial misperception of the object's orientation, as demonstrated in Experiment 1, in the error from the terminal position. An ANOVA of SOE data corrected for initial misperception (as for the above corrected OOE) showed no main difference in the results as compared with the initial SOE data. Therefore, errors on initial perception of object's orientation do not account for the distribution of errors observed here.

Finally, we found no significant correlation between the mental imagery test/questionnaires and the errors on object orientation updating from the subjective terminal position (SOE).

\section{GENERAL DISCUSSION}

\section{Effect of Processing Mode on (1) Locomotor Activity and (2) Response Latencies}

This is, to our knowledge, the first study to compare the effect of two distinct mental processing modes during guided nonvisual navigation following a visual preview of an object's location and orientation in a reduced-cue condition. In the OC task, observers continuously kept track of the object's perspective during the walk, whereas in the TC task, observers deduced its perspective at a final viewing position from continuous trajectory mapping and recall of the initial view on the object. In a preliminary experiment, in order to measure the error for initial perception of the object's orientation, observers rotated a model of the previously viewed object until it matched the memorized orientation of its counterpart object. Results showed that observers' estimates tended to be biased toward diagonal $\left(-45^{\circ} / 135^{\circ}\right.$ and $\left.45^{\circ} /-135^{\circ}\right)$ orientations of the object.

1. The self-paced locomotor movements were significantly slower in the OC task than in the TC task. Actually, in the latter processing mode, subjects mainly had to process the trajectory of their walk; that is, they only had to update the distance walked and the angle turned until the terminal position. In contrast, during the OC task, this updating of distance and angle had to be accompanied by a continuous updating of the object orientation and location itself, which imposed a much larger cognitive load on the subject and thus led to slower walking and turning velocities.

2 . The results of the walking experiment demonstrated that subjects take more time to retrieve information on object orientation in the TC task than in the OC task. In the OC task, since observers have continuously updated the object's perspective during their walk, the response is permanently available in the visuospatial buffer where image transformation is supposed to occur (Kosslyn, 1981, 1991). On the contrary, in the TC task, subjects were concerned only with mentally tracing the path they walked and refreshing the initial view of the object in memory. Consequently, from the terminal position, additional computations were required in order to determine the object's orientation response. These added at least $2.43 \mathrm{sec}$ to the response latency compared with the $\mathrm{OC}$ task.

\section{Effect of Processing Mode on the Updating of the Object's Location and Orientation}

The effects of the processing modes described above on the updating of the object's location and orientation were analyzed under the general assumption that observers correctly perceived their course while walking blindfolded. This is supported by the "rectilinear normalization" effect reported by Stevens and Coupe (1978), whereby navigation and orientation judgments tend to be carried out in (and distorted toward) a normalized, right-angle grid (Sadalla \& Montello, 1989), and also by postexperimental interview. It is also supported by Fujita et al.'s (1993) encoding-error model, fitted to data obtained from subjects who walked back to their initial location after a guided walk without vision on the two first segments of a triangular path.

We tested three main hypotheses on the origin of the updating errors to find out which one would account better for our results. In Hypothesis 1, it is assumed that subjects perceive correctly the distance walked, but make errors when turning toward the object to face it. Although this contradicts Haber et al. (1993), showing that the facing method is the more accurate method for this kind of localization response, we considered the facing performance as a noise not worth being taken into account for the analysis of the error on object's orientation. Therefore, we examined directly the difference between the orientation of the object estimated by the subject and the correct response from the current viewing position. Under this hypothesis, we observed an underrotation of the object 
orientation, increasing with the distance walked, as if the rate of transformation of the imagined object (rotation around its vertical axis) was less than for its corresponding physical counterpart. This result contradicts Pinker and Finke's (1980) demonstration that mental images rotating in depth (around the vertical gravity axis) "move" a constant fraction farther than the amount defined by the physical rotation of the real object, and thus seriously disputes Hypothesis 1 .

We also observed larger absolute errors on the object's orientation in the TC than in the OC task. These errors were due to misalignment and "mirror image" reversal errors typical of the use of directional information from a vantage point different from the one in which it was learned (Levine et al., 1982; Rossano \& Warren, 1989).

Then, we tested Hypothesis 2, which assumes that walked distance and estimation of final heading were correct, but subjects misperceived the object's location from the starting position. The object's location initially perceived and updated was computed by triangulating mean final headings obtained at each leg of the L-shaped path, in a fashion similar to that used by Fukusima et al. (in press). We triangulated headings equivalent in terms of the amount of head turn needed for facing the object correctly from the terminal position. Results showed that, under Hypothesis 2, the initial location of the object would have been different depending on terminal viewing position, which is not possible. If there was such an initial misperception of self-to-object distance, the triangulated object locations would have fallen onto viewer's initial line of sight (Philbeck \& Loomis, in press). However, triangulating the heading of the subjects from the endpoints of each path segment (i.e., Terminal Positions 3 and 6 ) led to subjective locations of the object that show a deviation lateral to the initial line of sight of the viewer-a result clearly inconsistent with Hypothesis 2.

Since the subjective object locations, under Hypothesis 2, were related to the walked distance, we reevaluated the data under the assumption that subjects made errors on the perception of the distance they walked, rather than on initial perception of the object's location. Accordingly, under Hypothesis 3, the subject's final facing direction (heading) toward the object location would reflect her/his subjective viewing position on the path, after accurate initial perception of object's location as well as correct execution of the intended head turn toward object location. Therefore, we shifted along the path the direction faced by the observers until it crossed the real location of the object, in order to compare the actual orientation-of-the-object response to the one that was expected from this subjective vantage position. The results, under Hypothesis 3, showed that the object was rotated a constant amount farther than expected from viewer's subjective position for terminal positions falling on the first segment of the L-shaped trajectory, which is consistent with previous research (Pinker \& Finke, 1980). However, on the subsequent segment, the errors dropped to around zero. This difference in performance suggests an effect of the $90^{\circ}$ turn between both segments, which was supposed to be perceived correctly in our assumptions for the different hypotheses. Nevertheless, the present residual error of SOE is already near zero; therefore, the much more complex model of error sources, including turn and distance misperception, is not reported here.

Finally, we found that the initial orientations of the object falling either on the observer's line of sight or perpendicularly to it were less prone to updating errors than were the other intermediate orientations.

In conclusion, estimation of walked distance and spatial inference processes (cf. in TC task when directional information is used from a vantage point different from the one in which it was learned) are the two main sources of errors for updating an object's location and orientation while walking blind under external guidance. This does not rule out the possibility of a supplementary source of error from encoding the object's location and orientation from an initial viewing position.

\section{Mental Imagery Tests and Questionnaires}

Using the Movement Imagery Questionnaire (MIQ; Hall \& Pongrac, 1983) and performance at a goal-directed locomotor pointing task, Corlett, Anton, Kozub, and Tardif (1989) found that accuracy at blind target-directed walking is not imagery dependent. However, since the MIQ assesses individual differences in visual and kinesthetic imagery of body segments movement, without requiring the subject to image locomotion movements (Hall, 1985; Hall, Pongrac, \& Buckholz, 1985), it may be inappropriate to use this specific questionnaire in the study of nonvisual navigation. Although it was already shown that many subjects with poor visual imagery (on a selfreport scale) have excellent spatial skills as evaluated by other tests (see Poltrock \& Agnoli, 1986, for a review), we wanted to extend this kind of correlational investigation to a more complex navigation task. We used TVIC on the basis that it includes items that are appropriate to the measurement of imagery control that involves the imagining of physical manipulation or movement (Morrison \& White, 1984). However, recent studies cast doubt on the sensitivity of both the VVIQ and TVIC (Kihlstrom, Glisky, Peterson. Harvey, \& Rose, 1991) to individual differences in imagery ability because these imagery questionnaires confuse the dimensions of vividness and control and fail to apply coherent definitions of either attribute of imagery (Kihlstrom et al., 1991; Lorenz \& Neisser, 1985). Finally, our failure to find any correlation between the TVIC, VVIQ, or MRT and the errors at object orientation updating from the viewer's subjective location establishes the limits of using mental imagery tests and questionnaires in order to predict or explain performance at nonvisual navigation.

\section{Topographic Disorders}

Neurological patients may display syndromes suggestive of a dissociation between modes of processing spatial information centered on objects or landmarks versus the walked trajectory. Persons suffering from constructive topographic agnosia (Grüsser \& Landis, 1991) due to le- 
sions in the lateral temporo-parieto-occipital lobe orient themselves well with respect to landmarks, but are unable to combine the different structures located in the extrapersonal space in the right order. According to the present findings, such patients have a deficit in implementing a TC mode of processing, and instead benefit more from using an OC mode of processing of their spatial representations, despite the higher on-line cognitive cost of the latter processing mode.

\section{A General Model}

Finally, congruent pieces of evidence in favor of a cooperation between both processing modes are supplied by research on multisensor integration modeling (Bernstein, 1967; Droulez \& Berthoz, 1991; Durrant-White, 1990 ) and autonomous mobile robot navigation (Brown, Durrant-White, Leonard, Rao, \& Steer, 1989). This research suggests that when one type of sensor information (e.g., vision) is missing, cooperative information will be supplied by other sensors in order to update an internal model of the missing sensor input. However, because of errors that increase over time, new visual input will be necessary periodically for resetting the internal model of the sensor input (Brown et al., 1989). Similarly, we suggest that during nonvisual navigation, available sensors cooperate to allow partial re-creation of the missing visual information through mental imagery. However, at certain steps, spatial inference (computational) processes would be necessary to allow reconstruction of the global perspective structure representation. During everyday life locomotion, we may therefore keep track of only part of the spatial layout during our displacement, and then regenerate the remainder from the new vantage points. Such an "updating-reconstruction-updating" cycle would constitute the basis of perspective structure updating during nonvisual navigation. Thus, further attention should be paid to the various processing modes adopted by subjects when updating temporarily nonvisually available spatial information.

In summary, we contend that until now the stages of processing fed by the path integration computations have been neglected in the literature on nonvisual navigation. It is certainly the case because the processing modes do not differ in the mean signed errors they produce. Our work is a definite invitation to use paradigms other than interference tasks in order to study the characteristics and effects of different processing modes on the updating of spatial information.

\section{REFERENCES}

Amorim, M.-A., Loomis, J. M., \& Fukusima, S. (1995). Spatial updating of object shape during real and imagined viewpoint change following visual preview. In B. G. Bardy, R. J. Bootsma, \& Y. Guiard (Eds.), Studies in perception and action III (pp. 217-220). Hillsdale, $\mathrm{NJ}$ : Erlbaum.

BerNaRD, J.-M. (1994). Introduction to inductive analysis. Mathématiques, Informatique, et Sciences Humaines, 126, 71-80.

BERNSTEIN, N. (1967). The coordination and regulation of movements. New York: Pergamon.
BööK, A., \& GÄRLING, T. (1980). Processing of information about location during locomotion: Effects of a concurrent task and locomotion patterns. Scandinavian Journal of Psychology, 21, 185-192.

Brown, C., Durrant-White, H., Leonard, J., Rao, B., \& Steer, B. (1989). Centralized and decentralized Kalman filter techniques for tracking, navigation, and control (Revised Tech. Rep. 277). Rochester, NY: University of Rochester.

Cochran, W. G., \& Cox, G. M. (1957). Experimental designs Toronto: Wiley.

Corlett, J. T., Anton, J., Kozub, S., \& Tardif, M. (1989). Is locomotor distance estimation guided by visual imagery? Perceptual \& Motor Skills, 69, 1267-1272.

CoRletT, J. T., Kozub, S., \& Quick, H. (1990). Memory for distance to be walked following interfering activities. Journal of Human Movement Studies, 18, 117-127.

Cutting, J. E., \& Vishton, P. M. (1995). Perceiving layout and knowing distances: The integration, relative potency, and contextual use of different information about depth. In W. Epstein \& S. J. Rogers (Eds). Perception of space and motion (pp. 69-117). San Diego: Academic Press.

Droulez, J., \& Berthoz, A. (1991). The concept of dynamic memory in sensorimotor control. In D. R. Humphrey \& H. J. Freund (Eds.), Motor control: Concepts and issues (pp. 137-161). London: Wiley.

DURRANT-White, H. F. (1990). Sensor models and multisensor integration. In I. J. Cox \& G. T. Wilfong (Eds.), Autonomous robot vehicles (pp. 73-89). New York: Springer-Verlag.

EASTON, R. D., \& ShOLL, M. J. (1995). Object-array structure, frames of reference, and retrieval of spatial knowledge. Journal of Experimental Psychology: Learning, Memory, \& Cognition, 21, 483-500.

FerRigno, G., \& PEDOTTI, A. (1985). ELITE: A digital dedicated hardware system for movement analysis via real-time TV signal processing. IEEE Transactions on Biomedical Engineering, 32, 943-950.

FuJiTA, N., KLATZKY, R. L., LOOMIS, J. M., \& GOLLEDGE, R. G. (1993) The encoding-error model of pathway completion without vision. Geographical Analysis, 25, 295-314.

Fukusima, S. S., Loomis, J. M., \& DA Silva, J. A. (in press). Visual perception of egocentric distance as assessed by triangulation. Journal of Experimental Psychology: Human Perception \& Performance.

Glasgow, J. I., \& Papadias, D. (1992). Computational imagery. Cognitive Science, 16, 355-394.

GolledGE, R. G. (1987). Environmental cognition. In D. Stokols \& I. Altman (Eds.), Handbook of environmental psychology (pp. 131174). New York: Wiley.

GoRDON, R. (1949). An investigation into some of the factors that favour the formation of stereotyped images. British Journal of Psychology, 39, 156-167.

GrüsSER, O.-J., \& LANDIS, T. (1991). Getting lost in the world: Topographical disorientation and topographical agnosia. In J. CronlyDillon (Ed.), Vision and visual dysfunction: Visual agnosias and other disturbances of visual perception and cognition (Vol. 12, pp. 411-430). London: Macmillan.

Haber, L., Haber, R. N., Penningroth, S., Novak, H., \& RadgowSKI, H. (1993). Comparison of nine methods of indicating the direction to objects: Data from blind adults. Perception, 22, 35-47.

HaLL, C. R. (1985). Individual differences in the mental practice and imagery of motor skill performance. Canadian Journal of Applied Sport Sciences, 10, 17-21.

Hall, C. R., \& Pongrac, J. (1983). Movement imagery questionnaire. London, $\mathrm{ON}$ : University of Western Ontario.

Hall, C. R., PongraC, J., \& Buckholz, E. (1985). The measurement of imagery ability. Human Movement Science, 4, 107-118.

Huttenlocher, J., \& Presson, C. C. (1973). Mental rotation and the perspective problem. Cognitive Psychology, 4, 277-299.

Kihlstrom, J. F., Glisky, M. L., Peterson, M. A., Harvey, E. M., \& RoSE, P. M. (1991). Vividness and control of mental imagery: A psychometric analysis. Journal of Mental Imagery, 15(3-4), 133-142.

KlatzKy, R. L., Loomis, J. M., Golledge, R. G., CicinelLi, J. G., DoHeRTY, S., \& Pellegrino, J. W. (1990). Acquisition of route and survey knowledge in the absence of vision. Journal of Motor Behavior, 22, 19-43. 
KOSSLYN, S. (1981). The medium and the message in mental imagery: A theory. Psychological Review, 88, 46-66.

KosSLYN, S. M. (1991). A cognitive neuroscience of visual cognition: Further developments. In R. H. Logie \& M. Denis (Eds.), Mental images in human cognition (pp. 351-381). Amsterdam: Elsevier.

Lalrent, M., \& Cavallo, V. (1985). Role des modalités de prise d'informations visuelles dans un pointage locomoteur [The role of visual input modality in a locomotor pointing task]. L'Année Psychologique, 85, $41-48$.

Levine, M., Jankovic, I. N., \& PaliJ, M. (1982). Principles of spatial problem solving. Journal of Experimental Psychology: General, 111, 157-175.

LindBerg, E., \& Gärling, T. (1983). Acquisition of different types of locational information in cognitive maps: Automatic or effortful processing? Psychological Research, 45, 19-38.

LoARER, E., \& SaVoyant, A. (1991). Visual imagery in locomotor movement without vision. In R. H. Logie \& M. Denis (Eds.), Mental images in human cognition (pp. 35-46). Amsterdam: Elsevier.

LOHMAN, D. F. (1988). Spatial abilities as traits, processes, and knowledge. In R. J. Sternberg (Ed.), Advances in the psychology of human intelligence (Vol. 4, pp. 181-248). Hillsdale, NJ: Erlbaum.

Loomis, J. M., Da Silva, J. A., Fujita, N., \& Fukusima, S. S. (1992). Visual space perception and visually guided action. Journal of Experimental Psychology: Human Perception \& Performance, 18, 906-921.

Loomis, J. M., Klatzky, R. L., Golledge, R. G., Cicinelli, J. G., PelLEGRINO, J. W., \& FRY, P. A. (1993). Nonvisual navigation by blind and sighted: Assessment of path integration ability. Journal of Experimental Psychology: General, 122, 73-91.

LORENZ, C., \& NeISSER, U. (1985). Factors of imagery and event recall. Memory \& Cognition, 13, 494-500.

MARKS, D. F. (1973). Visual imagery differences in the recall of pictures. British Journal of Psychology, 64, 17-24.

Mittelstaedt, H., \& Mittelstaedt, M. (1980). Homing by path integration in a mammal. Naturwissenschaften, 67, 566.

MitTelstaedt, M. L., \& Glasauer, S. (1991). Idiothetic navigation in gerbils and humans. Zoologische Jahrbucher: Abteilung für algemeine Zoologie und Physiologie der Tiere, 95, 427-435.

MorRISON, P. R., \& WhITE, K. D. (1984). Imagery control: What is really being measured? Journal of Mental Imagery, 8(2), 13-18.

Philbeck, J. W., \& Loomis, J. M. (in press). A comparison of two indicators of visually perceived egocentric distance under full-cue and reduced-cue conditions. Journal of Experimental Psychology: Human Perception \& Performance.
Pinker, S., \& Finke, R. A. (1980). Emergent two-dimensional patterns in images rotated in depth. Journal of Experimental Psychology: Human Perception \& Performance, 6, 244-264.

Poltrock, S. E., \& AGNoli, F. (1986). Are spatial visualization ability and visual imagery equivalent? In R. J. Sternberg (Ed.), $A d$ vances in the psychology of human intelligence (Vol. 3, pp. 255296). Hillsdale, NJ: Erlbaum.

RiESER, I. J. (1989). Access to knowledge of spatial structure at novel points of observation. Journal of Experimental Psychology: Learning, Memory, \& Cognition, 15, 1157-1165.

RIESER, J. J. (1990). Development of perceptual-motor control while walking without vision: The calibration of perception and action. In H. Bloch \& B. I. Bertenthal (Eds.), Sensory-motor organizations and development in infancy and early childhood (pp. 379-408). Amsterdam: Kluwer.

RIESER, J. J., \& RIDER, E. A. (1991). Young children's spatial orientation with respect to multiple targets when walking without vision. Developmental Psychology, 27, 97-107.

Rossano, M. J., \& WarRen, D. H. (1989). Misaligned maps lead to predictable errors. Perception, 18, 215-229.

RouAnET, H. (1996). Bayesian methods for assessing importance of effects. Psychological Bulletin, 119, 149-158.

Asserting the importance or the negligibility of effects with Bayesian methods. Psychological Bulletin.

Rouanet, H., \& Lecoutre, B. (1983). Specific inference in ANOVA: From significance tests to Bayesian procedures. British Journal of Mathematical \& Statistical Psychology, 36, 252-268.

Sadalla, E. K., \& Montello, D. R. (1989). Remembering changes in direction. Environment \& Behavior, 21, 346-363.

Stevens, A., \& Coupe, P. ( 1978). Distortions in judged spatial relations. Cognitive Psychology, 10, 422-437.

THORNDYKE, P. W., \& HAYES-RoTH, B. (1982). Differences in spatial knowledge acquired from maps and navigation. Cognitive Psychology, 14, 560-589.

TOYE, R. C. (1986). The effect of viewing position on the perceived layout of space. Perception \& Psychophysics, 40, 85-92.

VANDENBerG, S. G., \& Kuse, A. R. (1978). Mental rotations, a group test for three-dimensional spatial vizualization. Percepfual \& Motor Skills, 47, 599-604.

WAGNER, M. (1985). The metric of visual space. Perception \& Psychophysics, 38, 483-495.

(Manuscript received May 9, 1995; revision accepted for publication April 2, 1996.) 